\title{
A 12-week multi-component dietary intervention improves markers of non-alcoholic fatty liver disease in Irish patients in tertiary care
}

\author{
Deirdre Ní Fhloinn ${ }^{1}$, Ciara Wright ${ }^{2}$, Sara Naimimohasses ${ }^{3}$, Stephen Finn ${ }^{3}$, Suzanne Norris ${ }^{3}$, \\ Kath H. Hart ${ }^{1}$ and J. Bernadette Moore, \\ ${ }^{1}$ Department of Nutritional Sciences, University of Surrey, Guildford, Surrey, United Kingdom, \\ ${ }^{2}$ Glenville Nutrition, Dublin, Ireland, \\ ${ }^{3}$ Hepatology Department, St James Hospital, Dublin, Ireland and \\ ${ }^{4}$ School of Food Science and Nutrition, University of Leeds, Leeds, United Kingdom
}

\begin{abstract}
Non-alcoholic fatty liver disease (NAFLD) is a significant public health concern closely linked to obesity, affecting an estimated $25 \%$ of adults in Europe. Understudied in Ireland, the aim of this research was to examine the effects of a 12-week multi-component dietary intervention on weight loss and markers of liver injury in Irish NAFLD patients in tertiary care. Biopsy confirmed NAFLD patients $(n=27)$ were recruited from St James' Hospital in Dublin, Ireland. Consenting participants underwent a 12-week moderate-intensity intervention incorporating weekly group nutritional education, behavioural change and group support, as well as individualised advice and weigh-ins from a trained nutritionist. Control group participants were given routine clinical care. All participants were clinically reviewed before, immediately after, and 3 months post intervention. Individuals $(n=12)$ with histological evidence of steatohepatitis underwent a repeat liver biopsy on completion of the intervention. Detailed dietary assessment was performed using both a 4-day diet diary (4DDD) and a novel, recently validated, short food frequency questionnaire (SFFQ) designed specifically to assess habitual intakes of food items related to NAFLD. Nutrient intakes were analysed using myFood $24^{\mathrm{TM}}$ dietary analysis software, and the Mediterranean diet quality score (MDQS) was used to assess the overall change in dietary patterns. Of the 15 participants who completed the intervention, $80 \%(n=12)$ achieved a weight loss exceeding $5 \%$, with $47 \%(n=7)$ achieving $>7 \%$. There were significant improvements from baseline to week 12 in the intervention group for the majority of clinical parameters including HbA1c $(\mathrm{p}=0.0054)$, liver enzymes (ALT, $p=0.0108$; GGT, $p=0.0001)$ and transient elastography (kPA, $p=0.0308 ;$ CAP, $p=0.0081$ ). However, these results failed to maintain significance when analysed compared to controls. The overall dietary pattern was significantly improved after 12 weeks as assessed by the MDQS ( $p=0.03$ ), with no apparent compromise in micronutrient intake despite the energy reduction. Reductions in energy, saturated fat, carbohydrate and sugar intakes at 12 weeks, were maintained at three months follow up. Analysis of pre- and post-intervention liver biopsies in the intervention group demonstrated a clinically significant improvement in NAS score $(p=0.0273)$, attributable to reductions in hepatic steatosis $(p=0.0078)$. A significant correlation was observed between improvement in liver histology and change in sugar intake $(r=0.7534, p=0.0093)$. Although results were somewhat limited by small sample size, nutritional education achieved beneficial dietary changes that persisted after the intervention ceased. Notably, achieving reductions in sugar intakes may be particularly beneficial in reducing the severity of hepatic steatosis in Irish adults with NAFLD.
\end{abstract}

\section{Conflict of Interest}

There is no conflict of interest 